\title{
Impugning Randomness, Convincingly
}

\author{
Yuri Gurevich
}

Microsoft Research

John organized a state lottery, and his wife won the main prize. One may feel that the event of her winning isn't particularly random, but is it possible to convincingly impugn the alleged randomness, in cases like this, in a fair court of law? We develop an approach to do just that.

We start with a principle that bridges between probability theory and the real world. The principle is known under different names in the history of probability theory. It is often formulated thus: events of sufficiently small probability do not happen. This formulation of the principle is too liberal, however. Events of small probability happen all the time. The common way to tighten the principle is to restrict attention to predicted events of sufficiently small probability. Further, we better restrict attention to a single probabilistic experiment. If you repeat the experiment sufficiently many times then there is a good chance that the predicted event of small probability will happen.

The tightened principle plays an important role in statistics. It justifies Fisher's test of statistical significance. Here, the "null hypothesis" is the hypothesis that the given probability distribution is the actual distribution governing the realworld experiment in question, and the "critical region" is induced by the predicted event of small probability.

Our Bridge Principle is a liberalization of the tightened principle above in two directions. One is that the specification of the small-probability event does not have to be given ahead of time; it suffices that it is given independently from the observed outcome of the experiment. The other and bolder liberalization is that the specification may be implicit. It is the Bridge Principle that we use to impugn the alleged randomness in cases like the lottery.

Note that traditional probability theory does not even have the notion of random events. The bolder liberalization above is informed by information complexity theory (also known as Kolmogorov complexity theory and as algorithmic information theory). Unfortunately, traditional information complexity theory is not applicable to real-world scenarios like the lottery one. We use logical definability to generalize information complexity theory (in a way that makes much of the traditional machinery irrelevant).

The talk touches upon issues that have been hotly discussed in several scientific communities, and we finish with an expansive survey of the ocean of related work.

The talk reflects joint work with Grant Passmore of Cambridge University. 